\title{
Expression and self-assembly of Heterocapsa circularisquama RNA virus-like particles synthesized in Pichia pastoris
}

\author{
WU YuanZheng ${ }^{1,4}$, KIM Wonduck $^{2}$, KIM Si-Wouk $^{2}$, EOM Chi-Yong $^{3}$, YANG HeTong $^{4} \&$ \\ SHIN Hyun-Jae ${ }^{1 *}$ \\ ${ }^{1}$ Department of Chemical and Biochemical Engineering, Chosun University, Gwangju 501-759, Republic of Korea; \\ ${ }^{2}$ Department of Environmental Engineering, Chosun University, Gwangju 501-759, Republic of Korea; \\ ${ }^{3}$ Seoul Center, Korea Basic Science Institute, Seoul 136-713, Republic of Korea; \\ ${ }^{4}$ Biotechnology Center of Shandong Academy of Sciences, Jinan 250014, China
}

Received November 3, 2011; accepted March 8, 2012; published online April 10, 2012

\begin{abstract}
Heterocapsa circularisquama RNA virus (HcRNAV) is the first single-stranded RNA virus to be characterized that infects dinoflagellates. The ability of HcRNAV coat protein (HcRNAV CP) to self-assemble into virus-like particles (VLPs) in vitro suggested that heterologous expression was possible, and that the VLPs might be ideal nanocontainers for the targeted delivery of genes and chemicals. In this paper, we report the expression of a codon-optimized HcRNAV 109 CP gene in Pichia pastoris and the production of self-assembled HcRNAV VLPs using large-scale fermentation. The HcRNAV $109 C P$ gene was synthesized according to the codon preference of $P$. pastoris and cloned into a pPICZA vector. The recombinant plasmid pPICZA-CPsyns was transformed into P. pastoris by electroporation. The resulting yeast colonies were screened by PCR and analyzed for protein expression by SDS polyacrylamide gel electrophoresis. After large-scale fermentation, the yield of HcRNAV CPsyns reached approximately $2.5 \mathrm{~g} \mathrm{~L}^{-1}$ within $4 \mathrm{~d}$. The HcRNAV VLPs were purified using PEG precipitation followed by cesium chloride density gradient ultracentrifugation, and were subsequently analyzed using UV spectrophotometry and transmission electron microscopy. Fluorescence dye-labeled myoglobin was loaded into the cages of the HcRNAV VLPs and the encapsulation was confirmed by fluorescence spectroscopy. The results point to the possible utilization in pharmacology or nanotechnology of HcRNAV VLPs produced by $P$. pastoris fermentation.
\end{abstract}

Heterocapsa circularisquama RNA virus (HcRNAV), virus-like particles (VLPs), expression, self-assembly, Pichia pastoris

Citation: $\quad$ Wu Y Z, Kim W, Kim S W, et al. Expression and self-assembly of Heterocapsa circularisquama RNA virus-like particles synthesized in Pichia pastoris. Chin Sci Bull, 2012, 57: 3288-3293, doi: 10.1007/s11434-012-5125-z

Harmful algal blooms (HABs), also known as red tides, have had a negative impact on aquatic ecosystems and have increasingly become a threat to human and marine health [1,2]. Rapid increases in an algal population can lead to water discoloration, shading of submerged vegetation, disruption of food-web dynamics and oxygen depletion in the water. HABs are known to have damaged the fishing industry, and to have affected shoreline quality and local economies. The potent neurotoxins can concentrate in filterfeeding shellfish and poison human consumers [3-5]. Even non-toxic algae can be harmful when they amass in suffi-

*Corresponding author (email: shinhj@chosun.ac.kr) cient numbers. HABs occur in many regions around the world, including Scandinavia, the North Pacific, the Caribbean and the South Pacific [6,7].

The toxic or harmful phytoplanktons that cause HABs are commonly dinoflagellates, such as Alexandrium and Karenia [8]. Most dinoflagellates have a unique structure that includes a nucleus known as the dinokaryon within which the chromosomes are attached to the nuclear membrane. Many efforts have been made to control harmful algal blooms with little success [9]. Today, the biological control of HABs is considered to be feasible [10]. Viruses that are abundant in marine systems replicate rapidly and tend to be host-specific, suggesting that single algal species 
could be targeted $[11,12]$. Parasites also have the potential to control algal bloom species, but their specific role in this regard is largely unknown [13].

Heterocapsa circularisquama RNA virus (HcRNAV) is the first single-stranded RNA virus to be characterized that infects dinoflagellates [14]. H. circularisquama Horiguchi, first observed in Uranouchi Bay, Japan, is a harmful bloomcausing dinoflagellate that specifically kills bivalves [15]. HcRNAV particles are polyhedral with a diameter of approximately $30 \mathrm{~nm}$, and encapsulate a single positive-stranded 4.4 kb RNA genome. Two open reading frames (ORF-1 and ORF-2) were identified in the genome, ORF-2 coding for the viral coat protein [16]. HcRNAV targets and accumulates in the dinoflagellate nucleus. The virus clones have been divided into types CY and UA (HcRNAV109 and HcRNAV34, respectively), based on their host strain specificity [17].

The methylotropic yeast, Pichia pastoris, has been shown to be a suitable system for the heterologous expression of virus coat protein, which could then self assemble into viruslike particles (VLPs) in vitro. The successful examples include cowpea chlorotic mottle virus (CCMV), hepatitis B virus (HBV), and bacteriophage Qbeta [18-20]. Besides its ability to express foreign proteins at a high level, $P$. pastoris has been shown to have several advantages over other expression systems. The fermentation period was usually 4 $5 \mathrm{~d}$, compared to the plant hosts in which production took weeks [21]. The synthesized VLPs were soluble and able to self-assemble, while production in prokaryote hosts, such as E. coli, often results in insoluble inclusions [22].

In this paper, we report on the heterologous synthesis of HcRNAV 109 coat protein by $P$. pastoris. The successful large-scale fermentation and purification of the self-assembled HcRNAV VLPs suggest their potential application as nanocontainers. To our knowledge, this is the first paper to report the heterologous expression of HcRNAV.

\section{Materials and methods}

\subsection{Synthesis of $P$. pastoris codon-optimized HcRNAV 109 CPsyns}

The sequence of the HcRNAV 109 CP gene, GenBank accession [AB218609] [23], was redesigned to substitute amino acid codons that are seldom used in $P$. pastoris with those more frequently used [24]. Chemical synthesis of the new CP gene (CPsyns) was accomplished with GenScript, USA. Restriction sites for EcoR I (GAATTC) and Not I (GCGGCCGC) were added upstream and downstream, respectively, of the CPsyns gene. The plasmid pUC57-CPsyns was transformed into $E$. coli Top10 for amplification and preservation.

\subsection{Construction of the recombinant vector pPICZA- CPsyns}

The $P$. pastoris host strain GS115 (his4, histidine-requiring auxotroph) and the intracellular expression vector pPICZA were purchased from Invitrogen, USA. For the in vivo expression in $P$. pastoris, the $1.1-\mathrm{kb}$ CPsyns gene was retrieved from the pUC57 vector as an EcoR I/Not I fragment and cloned into the corresponding EcoR I and Not I sites of the Pichia integrative vector, pPICZA. The resultant vector pPICZA-CPsyns was then transformed into E. coli Top10 for its amplification.

\subsection{Transformation of $P$. pastoris and cultivation of HCRNAV 109 CPsyns}

The transformation and expression of the CPsyns gene in $P$. pastoris was performed using established procedures [25]. The recombinant plasmid pPICZA-CPsyns was linearized with $S a c$ I and subsequently used to transform $P$. pastoris GS115 by electroporation (Multiporator, Eppendorf, Germany). The transformed yeast cells were incubated in YPD agar containing Zeocin at $30^{\circ} \mathrm{C}$ for $2-3 \mathrm{~d}$. Because the CPsyns gene was integrated into the $5^{\prime}$ alcohol oxidase promoter (AOX1) locus on the Pichia chromosome, all transformants would be $\mathrm{His}^{+} \mathrm{Mut}^{+}$. The CPsyns gene in the transformed yeast cells was amplified with PCR using the CPsyns and AOX1 primers (data not shown).

Verified transformants were grown in a buffered glycerol-complex medium (BMGY) at $30^{\circ} \mathrm{C}$ for $16-20 \mathrm{~h}$. The yeast cells were harvested using centrifugation and resuspended in buffered methanol-complex medium (BMMY) at an optical density of 1.0 at $600 \mathrm{~nm}$ (about $5 \times 10^{7}$ cells $\mathrm{mL}^{-1}$ ). Incubation was continued for an additional $96 \mathrm{~h}$ at $30^{\circ} \mathrm{C}$ with $1 \%$ methanol induction every $24 \mathrm{~h}$. To determine the optimal harvest time after induction, $1 \mathrm{~mL}$ of the expression medium was withdrawn every $12 \mathrm{~h}$ and the protein expression levels were determined using SDS polyacrylamide gel electrophoresis (SDS-PAGE). Non-transformed P. pastoris GS115 and cells transformed with the expression vector pPICZA/GS115 were included as controls for the same induction process.

\subsection{Large scale fermentation of HcRNAV 109 CPsyns}

A positive transformant GS115-24, reliably expressing the HcRNAV CPsyns gene, was selected to scale-up CPsyns production in a 5-liter fermenter (Fermentec XP 50, Korea) using established protocols (Pichia Fermentation Process Guidelines, Invitrogen, USA). A total of $10 \%$ of the initial fermentation volume of GS115-24 in BMGY was inoculated into BMGY in the fermenter. One $\mathrm{mL}$ of the fermentation medium was withdrawn every $12 \mathrm{~h}$ to monitor cell growth using absorbance at $600 \mathrm{~nm}$. Coat protein expression levels were determined using SDS-PAGE. After the glycerol fedbatch phase and the methanol fed-batch phase, the culture was centrifuged to separate the cells from the supernatant. The harvested $P$. pastoris cells were placed on ice for further lysis, while protein concentration was analyzed using 
the Bradford protein assay.

\subsection{Purification of HcRNAV 109 VLPs}

A modified virus capsid purification procedure based on PEG precipitation and density gradient centrifugation was employed to purify the soluble assembled HcRNAV VLPs [26]. The Pichia cells gathered by centrifugation at $4000 \times g$ for $30 \mathrm{~min}$ were resuspended in five times (w/w) lysis buffer (50 $\mathrm{mmol} \mathrm{L}^{-1}$ sodium phosphate, $\mathrm{pH} 7.4,1 \mathrm{mmol} \mathrm{L}^{-1}$ PMSF, $1 \mathrm{mmol} \mathrm{L}{ }^{-1}$ EDTA, and 5\% glycerol) and disrupted by sonication (30 s on, $30 \mathrm{~s}$ off, 15 cycles, Vibra-cell VCX-750, Sonics, USA). Cell debris and insoluble material were removed by centrifugation at $10000 \times g$ for $30 \mathrm{~min}$, and PEG 6000 was added to the supernatant to a final concentration of $10 \%$. After overnight incubation at $4^{\circ} \mathrm{C}$, the solution was centrifuged at $10000 \times g$ for $30 \mathrm{~min}$ and the resulting pellet was resuspended in phosphate buffer $\left(10 \mathrm{mmol} \mathrm{L}^{-1} \mathrm{Na}_{2} \mathrm{HPO}_{4}\right.$, $10 \mathrm{mmol} \mathrm{L}^{-1} \mathrm{KH}_{2} \mathrm{PO}_{4}, \mathrm{pH}$ 7.2). The resuspended PEG pellet was loaded onto a 10\%-40\% cesium chloride gradient and centrifuged at $35000 \times g$ for $2 \mathrm{~h}$. The pellet was dissolved in the same phosphate buffer for further analysis.

\subsection{Analysis of purified HcRNAV 109 VLPs}

The purified HcRNAV VLPs were analyzed by UV spectrometry and transmission electron microscopy (TEM). Absorption at $260 \mathrm{~nm}$ and $280 \mathrm{~nm}$ was measured on a Scinco S-3100 UV spectrophotometer (Seoul, Korea), and the $A_{260}$ : $A_{280}$ ratio was calculated. TEM images of VLPs negativelystained with $2 \%$ (wt:vol) uranyl acetate were obtained with a CM30 electron microscope (FEI/Philips) operated at $200 \mathrm{kV}$ (Korea Basic Science Institute, Chunchon Center, Korea) [26].

\subsection{Encapsulation of fluorescence dye-labeled myoglobin}

Purified HcRNAV VLPs in PBS, obtained from Section 2.5, were dialyzed against three changes of disassembly buffer (0.05 mol L $\mathrm{L}^{-1}$ phosphate buffer, $0.15 \mathrm{~mol} \mathrm{~L}^{-1} \mathrm{NaCl}, 2$ mmol L ${ }^{-1}$ DTT, pH 8.0). Myoglobin was labeled with a fluorescent dye, Alexa Fluor 594, according to the Alexa Fluor ${ }^{\circledR} 594$ Protein Labeling Kit (A10239, Invitrogen, USA). The disassembled HcRNAV VLPs and fluorescent myoglobin were mixed at the ratio of $5: 1$ and the resulting mixture was incubated at room temperature for $1.5 \mathrm{~h}$. The incubated solution was then dialyzed against three changes of reassembly buffer $\left(0.05 \mathrm{~mol} \mathrm{~L}^{-1}\right.$ phosphate buffer, $0.5 \mathrm{~mol} \mathrm{~L}^{-1}$ $\mathrm{NaCl}, \mathrm{pH}$ 7.2) and concentrated by freeze-drying. Free myoglobin was removed using Vivaspin 6 ultrafiltration spin column (Sartorius, Germany). The VLPs containing encapsulated myoglobin were further analyzed and purified by size exclusion fast performance liquid chromatography (FPLC) on Biologic DuoFlow ${ }^{\mathrm{TM}}$ Chromatography System (Bio-Rad, USA) equipped with a Superose 6 PC 3.2/30 column (flow rate $40 \mu \mathrm{L} \mathrm{min}{ }^{-1}$, GE Healthcare, USA). The purified myoglobin-containing HcRNAV capsids were used to perform fluorescence spectroscopy (Confocal Laser Scanning Biological Microscope FV1000, Olympus, Japan) [27].

\section{Results and discussion}

\subsection{Construction of expression vector pPICZA-CPsyns}

The $P$. pastoris expression system was chosen due to its relatively simple fermentation protocol and high final cell density. To enhance the efficiency of gene expression, the HcRNAV CPsyns gene was optimized to the codon usage of $P$. pastoris (Figure 1). The synthesized 1080 bp sequence showed $95.6 \%$ nucleotide identity (1033/1080) and 100\% deduced amino acid identity with the native HcRNAV 109 $C P$ gene. The inclusion of seldom-used codons in P. pastoris, such as CGG (arginine) and GCG (alanine), can lead to low efficiency of gene translation via early termination. These codon alterations $(\mathrm{CGG} \rightarrow \mathrm{AGA}, \mathrm{GCG} \rightarrow \mathrm{GGT})$ were expected significantly to improve the expression level of foreign proteins. In total, 47 changes were introduced into the synthesized gene. The resulting HcRNAV CPsyns was then inserted into the pPICZA plasmid. The digestion of the recombinant vector by $E c o$ R I and Not I showed two bands of $3.3 \mathrm{~kb}$ (pPICZA) and $1.1 \mathrm{~kb}$ (CPsyns), which indicated that the expression vector pPICZA-CPsyns was constructed successfully (Figure 2).

\subsection{Expression of the HcRNAV 109 CPsyns gene in $P$. pastoris}

After screening on Zeocin-YPD plates, over 10 P. pastoris transformants were induced with methanol and examined for HcRNAV CPsyns production. Each supernatant $(5 \mu \mathrm{L})$ after cell lysis was subjected to SDS-PAGE (Figure 3). All of the transformants showed a band at approximately $40 \mathrm{kD}$, which was the theoretical molecular weight of the HcRNAV coat protein monomer. Transformant GS115-24 was selected for further fermentation.

The $P$. pastoris transformant GS115-24 grew exponentially for more than $40 \mathrm{~h}$ in the glycerol fed-batch phase in the 5-liter fermenter. Then, a methanol-fed induction was commenced over $72 \mathrm{~h}$ with the methanol concentration beginning at $3.6 \mathrm{~mL} \mathrm{~h}^{-1} \mathrm{~L}^{-1}$ and reaching $10.9 \mathrm{~mL} \mathrm{~h}^{-1} \mathrm{~L}^{-1}$. The maximum wet cell weight reached was $400 \mathrm{~g} \mathrm{~L}^{-1}\left(A_{600}\right.$ of approximately 160). The concentration of crude HcRNAV coat protein approached $2.5 \mathrm{~g} \mathrm{~L}^{-1}$ as determined by the Bradford protein assay, which was more than 12-fold the yield of HcRNAV CP in the shaking flasks. This is one of the highest productions of viral coat protein reported to date.

\subsection{Purification of HcRNAV 109 VLPs}

Recombinant GS115-24 cells were lysed by ultrasonic 


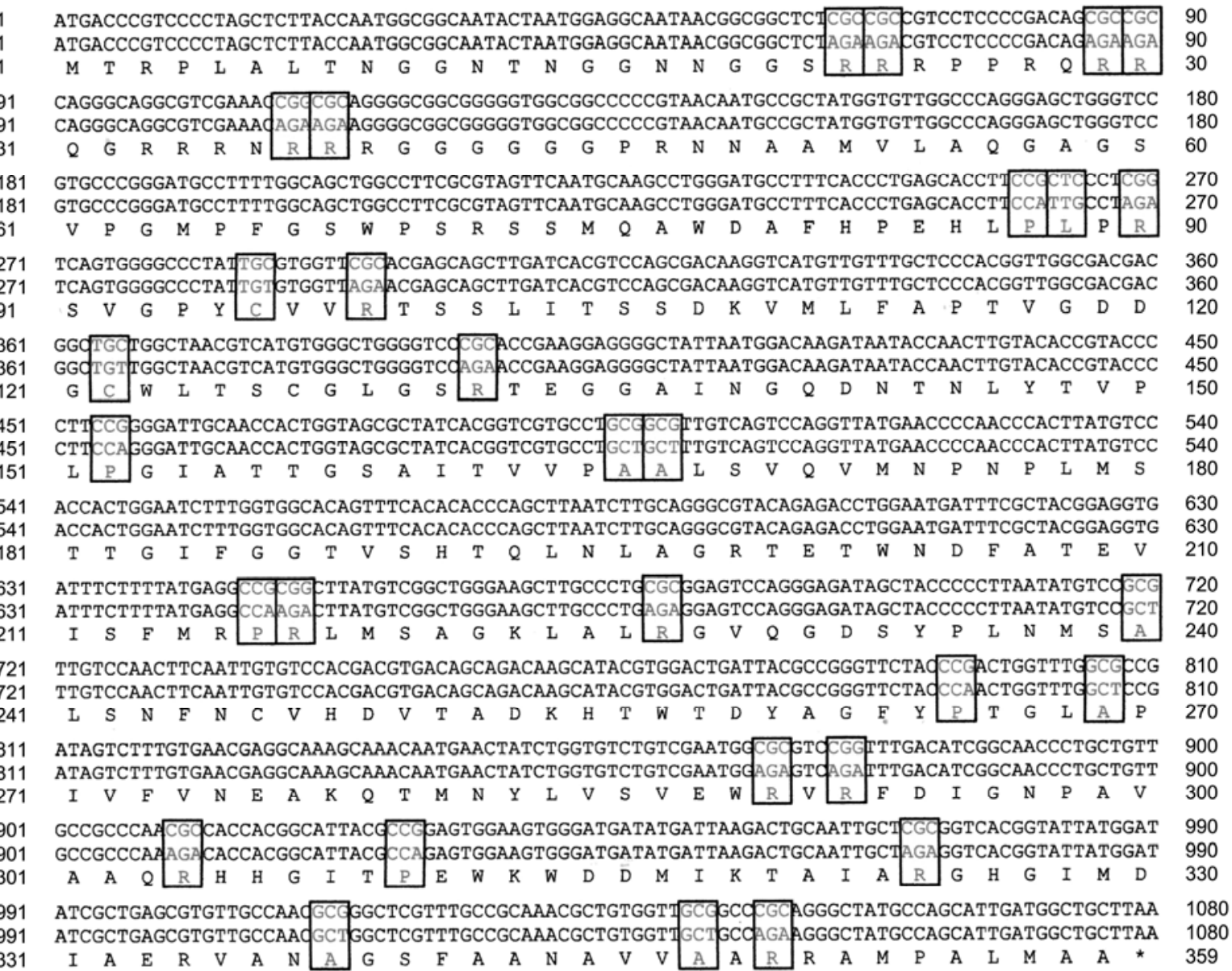

Figure 1 Nucleotide sequences and deduced amino acid sequence of HcRNAV 109 CP gene (top line) and HcRNAV CPsyns gene (bottom line). The alterations in nucleotide sequence are boxed in gray.

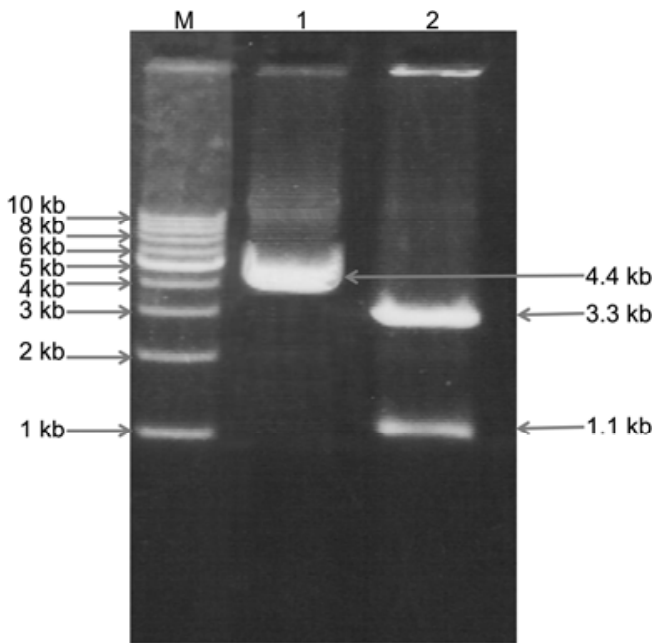

Figure 2 Digestion of pPICZA-CPsyns with EcoR I and Not I. Lane 1, digestion of pPICZA-CPsyns with EcoR I; 2, double-digestion of pPICZACPsyns with EcoR I and Not I; M, Takara $1 \mathrm{~kb}$ DNA ladder.

disruption and the HcRNAV CP purified by PEG precipitation and density gradient centrifugation. PEG treatment of the supernatant preferentially precipitated HcRNAV CP

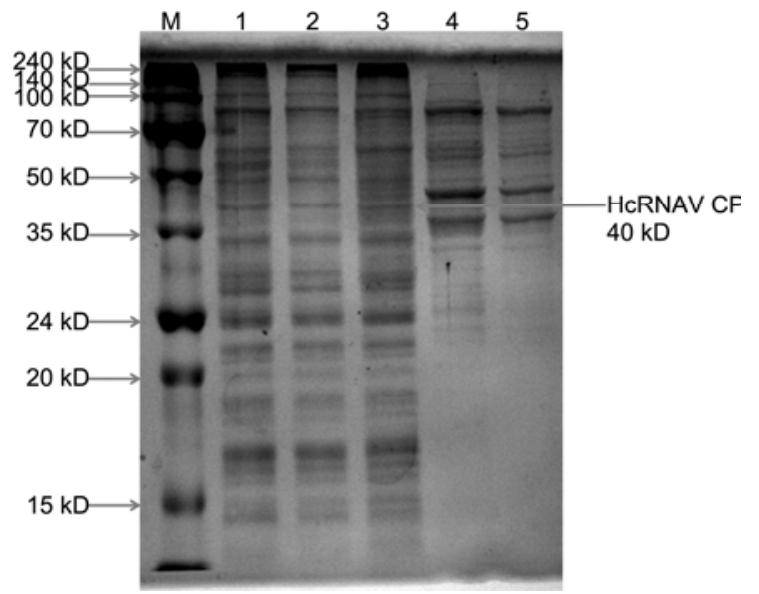

Figure 3 SDS-PAGE of $P$. pastoris transformants. Lanes 1-3, soluble fractions of pPICZA-CPsyns transformants after sonication; 4, control of pPICZA/GS115; 5, control of GS115; M, DokDo-MARK ${ }^{\mathrm{TM}}$ broad-range.

with almost no precipitation of other proteins. Ultracentrifugation in $\mathrm{CsCl}$ further purified HcRNAV CP to a single band of $40 \mathrm{kD}$, which showed that the coat protein was able to assemble into high molecular weight structures (HcR- 
NAV VLPs) (Figure 4).

\subsection{Characterization of purified HcRNAV 109 VLPs}

The VLPs were analyzed by UV spectrophotometry and showed an $A_{260}: A_{280}$ ratio of 1.51 , which was similar to the ratio of 1.56 observed with other VLPs.

TEM examination of the VLPs showed spherical particles of ca. $30 \mathrm{~nm}$ diameter (Figure 5). This similarity to native HcRNAV particles indicated that the synthesized coat protein was successfully expressed and self-assembled into VLPs in vitro [14].

\subsection{Encapsulation of fluorescence dye-labeled myoglobin}

The co-elution of HcRNAV VLPs and guest myoglobin $(t=$ 36 min) was observed in the FPLC traces (Figure 6), showing the same elution time as the original capsids. These pointed to the successful reassembly of the viral cage after the

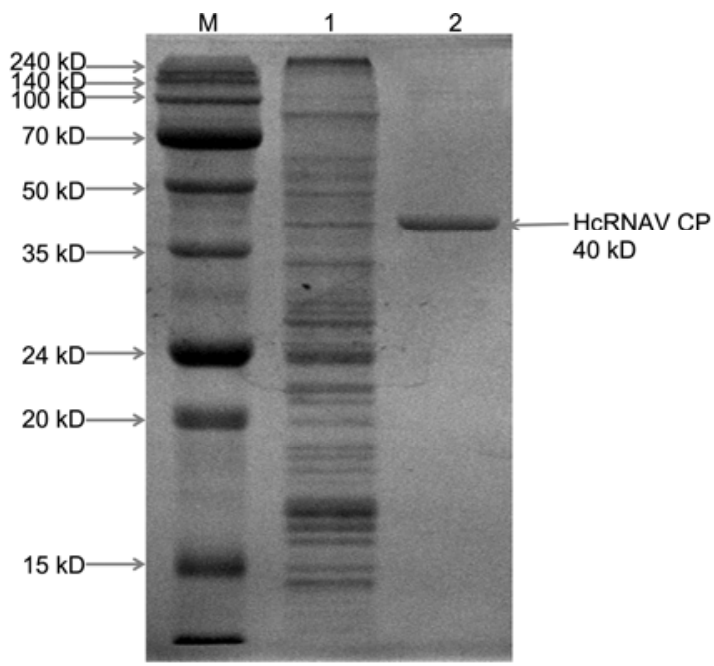

Figure 4 Purification of HcRNAV coat protein. Lane 1, soluble fractions of GS115-24 after sonication; 2, HcRNAV CP purified by ultracentrifugation in $\mathrm{CsCl}$; M, DokDo-MARK ${ }^{\mathrm{TM}}$ broad-range.

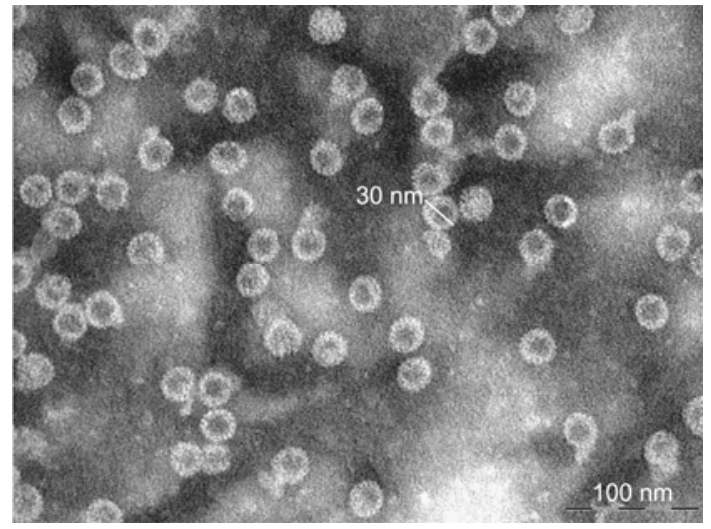

Figure 5 TEM image of HcRNAV VLPs. The diameter of the capsids was about $30 \mathrm{~nm}$. Size bar represents $100 \mathrm{~nm}$.

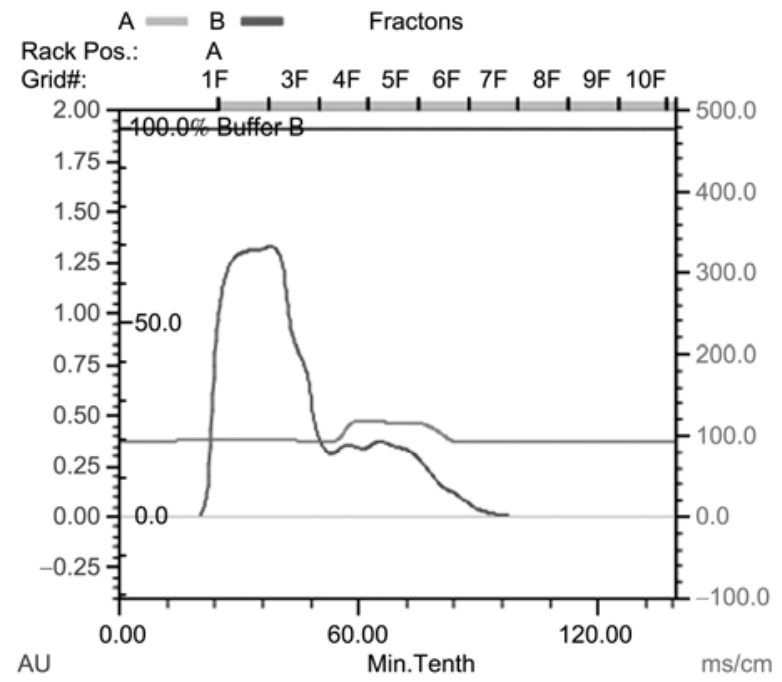

Figure 6 Size-exclusion FPLC of myoglobin-containing HcRNAV VLPs at $280 \mathrm{~nm}$. The peak at elution time of $36 \mathrm{~min}$ corresponded to the intact virus capsids.

encapsulation process.

The correct reassembly of HcRNAV capsids with fluorescent myoglobin was further proven by fluorescence spectroscopy. The capsids containing this guest protein displayed significant emission at $\lambda=590 \mathrm{~nm}$ (Figure 7), indicating that inclusion of myoglobin had occurred successfully. When HcRNAV VLPs were used as control, no emission under the same condition was observed.

\section{Conclusion}

HcRNAV 109 coat protein was expressed in Pichia pastoris

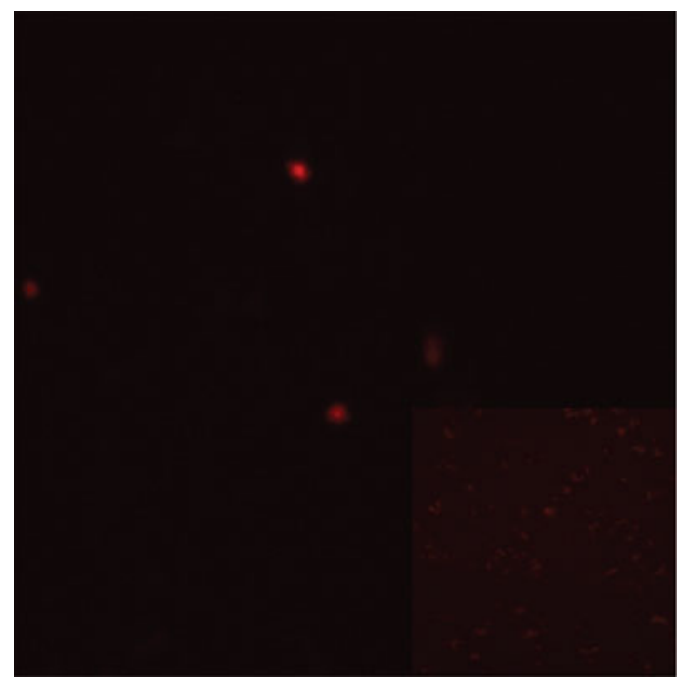

Figure 7 Fluorescence spectroscopy of myoglobin-containing HcRNAV VLPs. A typical confocal fluorescence image $(1.68 \mu \mathrm{m} \times 1.68 \mu \mathrm{m})$ showed the formation of a fluorescent protein encapsulated inside a capsid. Inset: AFM image (to scale) at the same sample location, showing that only a small fraction of the capsids contained myoglobin molecules. 
and successfully self-assembled into polyhedral virus-like particles. To our knowledge, this is the first report on the efficient expression and assembly in $P$. pastoris of the capsid of a virus infecting a harmful alga. Optimization of codons to those preferred by $P$. pastoris was necessary for the high expression of the foreign gene in the $P$. pastoris system [28]. The successful expression and purification of HcRNAV VLPs makes their abundant and rapid production now possible. This method also represents a promising tool for the preparation of large amounts of VLPs for use in gene therapy, vaccine development, and other applications such as the control of harmful algal blooms.

The encapsulation of myoglobin as a model protein inside HcRNAV VLPs revealed that these virus capsids derived from $P$. pastoris were suitable nanocontainers for drug delivery and imaging reagent carriers. The specific targeting by HcRNAV of $H$. circularisquama would be extremely useful in controlling harmful algal blooms. The encapsulation of algicidal compounds inside the capsids to improve the efficiency is another useful approach for future investigation. HcRNAV could become a model virus capsid that might be used as a nanoplatform in materials science and medicine. Further analysis to compare the properties of the recombinant HcRNAV capsids with those of the native types is ongoing. The modification of HcRNAV CP by altering certain functional amino acids could also be useful in revealing the detailed structure, currently unknown, of the HcRNAV capsid.

This work was supported by the Pioneer Research Center Program through the National Research Program of Korea funded by the Ministry of Education, Science and Technology, Korea (Grant M1071118001-08M1118-00110) (2010). We thank Dr. Song Her (KBSI) for providing the TEM pictures.

1 Anderson D M. Turning back the harmful red tide. Nature, 1997, 388 : 513-514

2 Hallegraeff G M. A review of harmful algal blooms and their apparent global increase. Phycologia, 1993, 32: 79-99

3 Van Dolah F M, Roelke D, Greene R M. Health and ecological impacts of harmful algal blooms: Risk assessment needs. Hum Ecol Risk Assess, 2001, 7: 1329-1345

4 Friedman M A, Levin B E. Neurobehavioral effects of harmful algal bloom (HAB) toxins: A critical review. J Int Neuropsychol Soc, 2005, 11: 331-338

5 Erdner D L, Dyble J, Parsons M L, et al. Centers for oceans and human health: A unified approach to the challenge of harmful algal blooms. Environ Health, 2008, 7(Suppl 2): 1-17

6 Corrales R A, Maclean J L. Impacts of harmful algae on seafarming in the Asia-Pacific areas. J Appl Phycol, 1995, 7: 151-162

7 Kirkpatrick B, Fleming L E, Squicciarini D, et al. Literature review of Florida red tide: Implications for human health effects. Harmful Algae, 2004, 3: 99-115

8 Garrett M, Wolny J, Truby E, et al. Harmful algal bloom species and phosphate-processing effluent: Field and laboratory studies. Mar Pollut Bull, 2010, 61: 621-626

9 Sengco M R, Anderson D M. Controlling harmful algal blooms through clay flocculation. J Eukaryot Microbiol, 2004, 51: 169-172

10 Solé J, Estradaa M, Garcia-Ladona E. Biological control of harmful algal blooms: A modelling study. J Marine Syst, 2006, 61: 165-179

11 Lawrence J E, Chan A M, Suttle C A. A novel virus (HaNIV) causes lysis of the toxic bloom-forming alga Heterosigma akashiwo (Raphidophycea). J Phycol, 2001, 37: 216-222

12 Baudoux A C, Brussaard C P. Characterization of different viruses infecting the marine harmful algal bloom species Phaeocystis globosa. Virology, 2005, 341: 80-90

13 Chambouvet A, Morin P, Marie D, et al. Control of toxic marine dinoflagellate blooms by serial parasitic killers. Science, 2008, 322: $1254-1257$

14 Tomaru Y, Katanozaka N, Nishida K, et al. Isolation and characterization of two distinct types of HcRNAV, a single-stranded RNA virus infecting the bivalve-killing microalga Heterocapsa circularisquama. Aquat Microb Ecol, 2004, 34: 207-218

15 Horiguchi T. Heterocapsa circularisquama sp. nov. (Peridiniales, Dinophyceae): A new marine dinoflagellate causing mass mortality of bivalves in Japan. Psychol Res, 1995, 43: 129-136

16 Nagasaki K, Shirai Y, Takao Y, et al. Comparison of genome sequences of single-stranded RNA viruses infecting the bivalve-killing dinoflagellate Heterocapsa circularisquama. Appl Environ Microbiol, 2005, 71: 8888-8894

17 Mizumoto H, Tomaru Y, Takao Y, et al. Intraspecies host specificity of a single-stranded RNA virus infecting a marine photosynthetic protist is determined at the early steps of infection. J Virol, 2007, 81: 1372-1378

18 Brumfield S, Willits D, Tang L, et al. Heterologous expression of the modified coat protein of cowpea chlorotic mottle bromovirus results in the assembly of protein cages with altered architectures and function. J Gen Virol, 2004, 85: 1049-1053

19 Hu B, Liang M J, Hong G Q. Expression of hepatitis B virus S gene in Pichia pastoris and application of the product for detection of antiHBs antibody. J Biochem Mol Biol, 2005, 38: 683-689

20 Freivalds J, Dislers A, Ose V, et al. Assembly of bacteriophage Qbeta virus-like particles in yeast Saccharomyces cerevisiae and Pichia pastoris. J Biotechnol, 2006, 123: 297-303

21 Yokoyama S. Protein expression systems for structural genomics and proteomics. Curr Opin Chem Biol, 2003, 7: 39-43

22 Zhao X, Fox J M, Olson $\mathrm{N} \mathrm{H}$, et al. In vitro assembly of cowpea chlorotic mottle virus from coat protein expressed in Escherichia coli and in vitro-transcribed viral cDNA. Virology, 1995, 207: 486-494

23 Nagasaki K. Dinoflagellates, diatoms and their viruses. J Microbiol, 2008, 46: 235-243

24 Sreekrishna K, Brankamp R G, Kropp K E, et al. Strategies for optimal synthesis and secretion of heterologous proteins in the methylotrophic yeast Pichia pastoris. Gene, 1997, 190: 55-62

25 Sambrook J, Russell D W. Molecular Cloning: A Laboratory Manual, 3rd ed. Cold Spring Harbor: Cold Spring Harbor Laboratory Press, 2001

26 Ali A, Roossinck M J. Rapid and efficient purification of cowpea chlorotic mottle virus by sucrose cushion ultracentrifugation. J Virol Methods, 2007, 141: 84-86

27 Comellas-Aragonès M, Engelkamp H, Claessen V I, et al. A virusbased single-enzyme nanoreactor. Nat Nanotechnol, 2007, 10: 635639

28 Macauley-Patrick S, Fazenda M L, McNeil B, et al. Heterologous protein production using the Pichia pastoris expression system. Yeast, 2005, 22: 249-270

Open Access This article is distributed under the terms of the Creative Commons Attribution License which permits any use, distribution, and reproduction in any medium, provided the original author(s) and source are credited. 